Cahiers « Mondes anciens »

ANCIENS

Histoire et anthropologie des mondes anciens

$6 \mid 2015$

Mères grecques

\title{
Le statut des mères dans l'Athènes classique
}

Motherhood as Status in Classical Athens

\section{Aurélie Damet}

\section{(2) OpenEdition}

Journals

Édition électronique

URL : http://journals.openedition.org/mondesanciens/1379

DOI : $10.4000 /$ mondesanciens. 1379

ISSN : 2107-0199

\section{Éditeur}

UMR 8210 Anthropologie et Histoire des Mondes Antiques

\section{Référence électronique}

Aurélie Damet, "Le statut des mères dans l'Athènes classique », Cahiers « Mondes anciens » [En ligne] 6 | 2015, mis en ligne le 16 février 2015, consulté le 01 mai 2019. URL : http://

journals.openedition.org/mondesanciens/1379; DOI : 10.4000/mondesanciens. 1379

Ce document a été généré automatiquement le 1 mai 2019.

\section{(c) $(1) \&$}

Les Cahiers «Mondes Anciens » sont mis à disposition selon les termes de la licence Creative Commons Attribution - Pas d'Utilisation Commerciale - Pas de Modification 4.0 International. 


\title{
Le statut des mères dans l'Athènes classique
}

\author{
Motherhood as Status in Classical Athens
}

\section{Aurélie Damet}

1 Si l'on suit les conclusions de Jean-Pierre Vernant (1987) sur le processus d'individuation à l'œuvre dans la Grèce classique, deux sphères en auraient permis l'émergence: la religion et le droit. C'est ce dernier prisme qui me permettra d'interroger ici la question de l'existence d'un statut de la mère dans l'Athènes classique ${ }^{1}$. D'après les traces juridiques laissées par la cité attique, est-il possible de déceler assez de prédispositions légales édifiant un sujet de droit autour de la figure maternelle? D'ores et déjà, s'interpose l'épineux et touffu buisson des controverses autour du statut juridique des femmes, catégorie dans laquelle on pourrait de prime abord penser le statut des mères. Les anciennes et récentes études sur la question statutaire dans le monde grec isolent le cas générique et très général des "femmes", mais ne proposent guère de réflexion précise sur les mères (Kamen 2013). Quant au dernier ouvrage en date sur les mères, il ignore le prisme juridique et légal (Petersen et Salzman-Mitchell 2012). Il y a pourtant de nombreux éléments qui, selon la définition large des statuts proposée par Moses Finley², permettent d'isoler un statut maternel : une série de droits et de privilèges acquis par la maternité, mais aussi des règles d'alliance qui concernent la figure maternelle. Pourtant, si les interrogations des historiens du droit et de la famille privilégient la figure paternelle et son rôle indéniable dans la fabrique du citoyen (Gherchanoc 1998 ; Bonnard 2003 ; Cantarella 2010 et 2011), il y a encore peu de place pour la reconnaissance du statut maternel.

\section{Préliminaires juridiques}

2 Comme souvent, les catégories contemporaines peuvent aider à penser les sociétés antiques et, dans le cas qui nous intéresse, aider à déterminer le statut juridique des mères athéniennes que l'on pourrait d'ores et déjà définir ainsi : dans la sphère citoyenne, 
les mères athéniennes ont bien une personnalité juridique, dans le sens où elles sont titulaires de droits. En revanche, il est plus difficile de percevoir les obligations des mères: c'est peut-être hors de la sphère légale que se définit le rôle attendu d'une génitrice, comme on le verra. Outre leur personnalité juridique dont les contours restent à définir, les mères athéniennes cumulent une capacité de jouissance et une incapacité d'exercice, stigmate de leur exclusion de la sphère politique institutionnelle, les privant d'un accès direct aux tribunaux et aux organes décisionnels de la cité. Pour l'écrire autrement, la mère athénienne peut jouir de certains droits qui lui sont conférés par sa qualité de parent mais, en tant que femme, elle ne peut défendre ces droits sans l'intermédiaire d'un représentant légal, le fameux kurios. Ce dernier n'est pourtant pas toujours indispensable car la cité athénienne a mis en place une protection directe des populations fragiles, pupilles, épiclères, veuves enceintes et parents âgés (AdamMagnissali 2007; Adam-Magnissali et Cudjoe 2010). L'archonte éponyme veille ainsi à punir tout outrage à une future mère isolée ([Aristote], Constitution d'Athènes, LVI, 7 ; Démosthène, XLIII, Contre Macartatos, 75) et la mère vieillissante peut, de son côté, profiter de l'existence de procès publics, graphai mais aussi eisangélie (Avotins 2004), qui instaurent une médiation et une surveillance par la collectivité. Le mélange d'infériorité statutaire et de possession de certains droits s'explique ainsi par le croisement nécessaire des paramètres de sexe, d'ancienneté et de fonction sociale et symbolique attribuée par la société athénienne aux femmes-mères, filles et épouses de citoyens.

\section{La mère athénienne, sujet de droits}

3 On peut déceler dans la législation attique une série de dispositions juridiques prenant pour objet la mère : il s'agit ou bien de la protéger ou bien de la placer au cœur de la légitimité citoyenne. Un premier ensemble d'exemples rappellera donc que toute génitrice peut attendre de la part de son fils un respect précisément encadré par la loi, reflet légal de lois plus coutumières et non écrites.

\section{Réflexions autour de la gerotrophia}

4 Parmi les obligations liées au respect des géniteurs, la gerotrophia occupe une place particulière dans les sources (De Schutter 1991; Leão 2011; Damet 2012, p. 217-219). Écoutons Démosthène à la tribune athénienne :

Aussi bien, je ne vois pas que, dans les familles, l'homme dans la force de l'âge traite ainsi les vieillards, ni qu'il se rencontre personne d'assez ingrat et absurde pour oser dire que, si tous n'en font pas autant que lui, lui-même ne fera rien. Un tel homme tomberait sous le coup des lois contre les mauvais traitements. Celles-ci n'exigent-elles pas, en effet, que le secours aux parents (goneûsi), imposé à la fois par la nature et par la loi, soit fourni consciencieusement et de bon cœur? (Démosthène, $\mathrm{X}, \mathrm{IV}^{\mathrm{e}}$ Philippique, 40) ${ }^{3}$.

5 Le générique « parent » comprend ici père et mère, auxquels il faut cependant ajouter les ascendants directs précisés par Isée :

Aux termes de la loi on doit nourrir ses ascendants (trephein tous goneas). Les ascendants sont le père, la mère, le grand-père, la grand-mère, le bisaïeul et la bisaïeule, s'ils vivent encore. L'ascendance remonte jusqu'à eux et les biens de tous ceux que j'ai énumérés sont transmis à leurs descendants. Aussi est-on tenu de les nourrir, même s'ils n'ont aucune fortune à laisser. Où serait la justice si nous 
encourrions une poursuite pour mauvais traitements (kakôseôs) en négligeant des parents alors même qu'ils ne devraient laisser aucune fortune, et si, au cas où ces mêmes parents en laisseraient une, notre adversaire en devait hériter et non pas nous ? La justice, à coup sûr, n'existerait plus (Isée, VIII, Succession de Kiron, 32-34).

6 Cet extrait d'Isée montre très bien que la mère est, comme les autres femmes de l' anchisteia, un maillon possible pour la transmission des héritages. C'est une des preuves parmi d'autres du caractère cognatique de la parenté athénienne (Wilgaux 2000 ; Roux 2009), où parentèles maternelle et paternelle ont un rôle reconnu. Cette bilinéarité en termes de système de parenté ne doit cependant pas occulter deux phénomènes qui nuancent l'apparente égalité entre père et mère. Tout d'abord le substrat idéologique de la supériorité masculine, qui traverse bon nombre de sources de l'Athènes classique et relègue symboliquement la mère à un rôle passif et secondaire dans l'engendrement des enfants (Bonnard 2004 ; Damet 2014), et ce malgré la loi de Périclès de 451. Mais aussi le fonctionnement même de l'anchisteia qui, en cas par exemple d'héritage à percevoir, privilégie les chaînons masculins et paternels par rapport aux chaînons féminins et maternels (Isée, XI, Succession d'Hagnias, 1-3 ; VII, Succession d'Apollodore, 18-20. Voir Vial 1985, p. 48 ; Just 1989, p. 83-89 ; Wilgaux 2000, p. 667 ; Damet 2012, p. 135-145), et, dans le cas de la captation d'une épiclère ${ }^{4}$, exclut même la parentèle maternelle de la jeune fille.

7 La gerotrophia est donc imposée par le droit positif attique mais, en tant que manifestation de gratitude (charis) envers les parents ${ }^{5}$, elle figure aussi au rang des agraphoi nomoi, les lois non écrites, ce que Démosthène entend par l'expression « [imposé] par la nature » (tês phuseôs). Un long passage des Mémorables de Xénophon (II, 2) présente ainsi un dialogue entre Socrate et son fils Lamproklès auquel le philosophe explique l'importance du respect moral et légal qu'il doit éprouver envers sa mère, même si celle-ci présente le caractère d'une vieille chouette athénienne ${ }^{6}$. Ce dialogue poursuit notamment le but de réhabiliter la figure socratique, accusée d'inciter les enfants à s'émanciper de l'autorité parentale et à retirer leur philia à des parents jugés inutiles, thématique traitée dans le dialogue du Lysis (Dorion 2006; Damet 2012, p. 42-47). Socrate part du principe que, depuis sa naissance, la mère de Lamproklès lui a toujours été utile et que ce dernier doit donc lui manifester de la gratitude sans condition; d'après Diogène Laërce, Socrate aurait réussi à convaincre son fils de ravaler sa colère contre sa mère et de l'honorer comme il se doit ${ }^{7}$. En ceci, l'Athénien n'est pas une exception historique et géographique, car la piété qu'un enfant doit manifester envers ses parents est un topos universel, autant dans la Grèce classique que dans les autres sociétés antiques et contemporaines (Xénophon, Cyropédie, VIII, 3, 49. Voir De Schutter 1991; Azoulay 2004, p. 45). À Athènes, par exemple, dans le cas où le fils meurt à la guerre, la cité se substitue à lui et veille aux besoins des parents survivants (Lysias, Oraison funèbre, 75b ; Platon, Ménexène, 248e-249c). Cependant la générosité de l'État athénien s'arrêtait là et seuls les parents des soldats morts pour la patrie bénéficiaient de cette caisse de pension. De plus, la gerotrophia athénienne pèse autant sur les fils biologiques que sur les fils adoptifs (Isée, III, Succession de Pyrrhos, 46; voir Rubinstein 1993), néanmoins la loi n'impose rien aux filles ${ }^{8}$ : dans les cas précis des mères, comme elles n'ont pas de recours possible à l'adoption, elles ne peuvent être prises en charge que par des fils biologiques. Enfin, l'obligation de nourriture n'est pas légalement imposée aux enfants bâtards, les nothoi (Plutarque, Vie de Solon, XXII, 4).

8 Cette dernière précision pourrait laisser penser que le respect des liens familiaux est corrélé à leur reconnaissance sociale. Toutefois cette impression doit être nuancée à la lumière de mythes comme celui d'ÆEdipe : en dépit d'une absence de reconnaissance du petit ÆEdipe par son père Laïos et malgré le défaut d'intégration rituelle au foyer de ce 
dernier et de Jocaste, c'est pourtant bien une atteinte au statut de père et de mère qu'CEdipe opère par le parricide et l'union incestueuse. Le statut parental conféré par le partage d'un même sang, de la mère comme du père, préexiste donc à la reconnaissance sociale de celui-ci, et c'est pourquoi le respect des parents figure finalement parmi les agraphoi nomoi, à très forte connotation religieuse. La famille grecque, et les études récentes sur la parenté insistent sur cet aspect, est bien un mélange de droit, de sang et de piété, pour reprendre les termes de Platon (Platon, Lois, 729c. Voir Wilgaux 2005a ; Brulé 2006 ; Morgan 2011 ; Damet 2012, p. 57-60 ; Gherchanoc 2012).

Il est intéressant de noter encore que le droit athénien prévoit des dérogations à l'obligation de la gerotrophia, mais uniquement en ce qui concerne le père. Ainsi, si un père livre son fils à la débauche et à la prostitution, il ne peut pas s'attendre, dans sa vieillesse, à être pris en charge par son enfant, qui ne lui devra que les funérailles (Démosthène, XXV, Contre Aristogiton I, 54, 58 et Dinarque, Contre Aristogiton, 8, 11, 14, 18). Plutarque ajoute que Solon aurait prévu que le père, qui se soustrait au droit à l'alimentation de ses propres enfants et au droit à l'éducation par l'apprentissage d'un métier, ne puisse en retour exiger la gerotrophia (Plutarque, Vie de Solon, XXII, 1. Voir Adam-Magnissali 2007, p. 157 ; Ruschenbusch 2010, p. 120-124). Ainsi, la vie, l'éducation et la subsistance sont les trois grands bienfaits donnés aux enfants par les parents (Xénophon, Les Mémorables, II, 2, 3-6), pour lesquels ils sont en droit d'attendre une gratitude à la fois naturelle et imposée par la loi.

$10 \mathrm{Si}$, ainsi, le droit athénien nous renseigne sur ce que peut être un mauvais père ne remplissant pas les devoirs que ses enfants et la société attendent de lui, ce même droit, alors qu'il attribue bien à la mère des privilèges, ne dit pas ce qu'est une bonne ou une mauvaise mère ${ }^{9}$. Si l'on reste dans le registre de la trophê, de nombreuses sources indiquent bien que la mère a un rôle attendu dans l'éducation et les soins quotidiens de l'enfant. Mais l'allaitement maternel, s'il est bien une coutume et un idéal social ${ }^{10}$, n'excluant pas le recours parallèle à des nourrices ou des biberons (Dubois 2012), n'est pas une obligation légale comme l'est la paidotrophia imposée au père. Le défaut de nourriture se présente cependant comme une composante du portrait de la mauvaise mère tragique qu'est Clytemnestre ${ }^{11}$ : Électre déplore de trouver vides les tables du palais ${ }^{12}$, et l'Oreste eschyléen n'a pas été allaité par sa mère, ce qui pourrait constituer une explication au contenu culpabilisant du fameux songe de la reine et éclairer le portrait psychologique de Clytemnestre ${ }^{13}$. À défaut d'encadrer les devoirs maternels, le droit athénien est bien plus précis sur le statut conjugal des femmes : en cas d'adultère avéré, les dispositions sur la moicheia prévoient que l'épouse soit répudiée et interdite de paraitre dans les fêtes publiques; la contrevenante peut être molestée par tout citoyen ${ }^{14}$. Il y a donc une nette distinction à faire au cours de la vie d'une femme athénienne, entre les statuts de mère et d'épouse, ce qui renvoie à la distinction plus générale entre la parenté par l'être (la consanguinité) et la parenté par le faire (l'alliance), qui n'induisent pas les mêmes droits et devoirs.

11 L'Athénien doit à ses parents un respect unanimement reconnu par le droit, la société, les individus et les dieux : l'atteinte à la mère comme au père, figures à « honorer à l'égal des dieux » (Eschine, I, Contre Timarque, 28), apparaît comme une violence scandaleuse et largement conspuée, à tel point que la sanction d'un tel comportement est minutieusement prévue par la législation athénienne, par le biais d'une procédure particulière, la graphê kakôseôs goneôn. 


\section{Mêtraloias et graphê kakôseôs goneôn}

12 Là encore, le délit de la kakôsis goneôn englobe autant la mère que le père comme potentielles victimes ${ }^{15}$. Ce forfait est traité par le biais d'une graphê qui peut être intentée par n'importe quel citoyen athénien (ho boulomenos). Contrairement aux autres graphai concernant les affaires de famille, tutelle d'orphelins et épiclérat (voir Harris 1999), la graphê kakôseôs goneôn n'entraîne pas de pénalité (azemios), sous forme d'amende de mille drachmes, dans le cas où celui qui intentait l'action ne remportait pas un cinquième des suffrages ${ }^{16}$. L'esprit de la loi est ici d'encourager au maximum la traque des mauvais fils par la communauté citoyenne et ce sans redouter un possible acquittement. Le paradoxe de ce délit est d'être un sujet récurrent dans les plaidoiries sans avoir pour autant laissé de traces effectives de poursuites avérées contre un individu. On retrouve donc souvent la mention de la kakôsis goneôn à des fins rhétoriques pour peindre le triste portrait d'un adversaire.

13 La kakôsis goneôn couvre plusieurs délits à l'encontre des ascendants: le refus de nourriture (mê trephein) [Xénophon, Les Mémorables, II, 2, 13 ; Eschine, I, Contre Timarque, 13; Démosthène, XXIV, Contre Timocrate, 107] et de logement (mê parechein oikêsin) [Eschine, I, Contre Timarque, 13], mais aussi les coups portés aux parents (tuptein) ${ }^{17}$, et le non-respect des devoirs funéraires (tous taphous mê kosmein) [Xénophon, Les Mémorables, II, 2, 13; Lycurgue, I, Conte Léocrate, 147]. Les individus convaincus d'un tel délit encourrent la perte des droits civiques, une atimie partielle sans confiscation des biens, l'interdiction de prendre la parole à l'Assemblée, de déambuler sur l'agora et de briguer une magistrature (Voir Solon fr.104b Ruschenbusch = Diogène Laërce, I, 55 ; Démosthène, XXIV, Contre Timocrate, 103 ; Eschine, I, Contre Timarque, 28-32 ; Andocide, I, Sur les Mystères , 74. Voir Damet 2012, p. 219-224). Lysias (XIII, Contre Agoratos, 91) et Démosthène (XXXIX, Contre Boeotos I, 33) vont jusqu'à évoquer la peine de mort. Le fils suspecté d'être ingrat ou violent envers son père ou sa mère prend le nom juridique de patraloias et de mêtraloias. Mais, si la cité encourage bien à dénoncer les mauvais garçons, elle ne permet pas qu'on calomnie un individu sur la base d'une grave accusation de kakôsis goneon. Ainsi, ces termes mêmes de mêtraloias et de patraloias figurent parmi la liste des mots interdits à fin diffamatoire, les apporhêta : celui qui abusait d'une telle accusation pour insulter un autre citoyen était passible d'un procès, la dikê kakêgorias (Damet 2012, p. 332-338; Azoulay et Damet 2014 ; Larran 2014).

14 L'absence d'amende pour utilisation abusive de la graphê kakôsis goneôn, les peines prévues à l'issue de cette accusation publique et enfin la mention des garnements parmi les aporrhêta montrent bien que la cité athénienne considérait l'atteinte aux parents comme un délit particulièrement sérieux, dépassant le strict cadre de l'oikos. Ce même principe d'imbrication entre sphère privée et sphère publique s'applique au cas des docimasies, examens préliminaires obligatoires, notamment pour briguer une magistrature athénienne, et qui délivrent un « certificat de bonnes mœurs ».

\section{La mère dans les docimasies}

À Athènes, parmi les docimasies politiques, quatre examens de contrôle se préoccupent particulièrement des relations familiales et privées de l'impétrant : celui des magistrats, des archontes, des bouleutes et des orateurs (Gagliardi 2005; MacDowell 2005; Feyel 
2009 ; Damet 2012, p. 404-408). Bon citoyen (voir Christ 2006), selon une liste de critères bien précis, le candidat doit être patriote et en règle avec le fisc, en plus d'être un bon fils (Dinarque, II, Contre Aristogiton, 17 ; [Aristote], Constitution d'Athènes, XLV, 3 ; LV, 2-3 ; Eschine, I, Contre Timarque, 28). Le respect de la mère réapparait ainsi dans les différentes docimasies, ainsi que Socrate le rappelle encore à son fils Lamproklès :

Alors tu serais prêt à te soucier de ces gens-là (les voisins, les compagnons de voyage ou de navigation) mais à ta mère, qui t'aime plus que personne, tu ne daignes pas prodiguer tes soins? Ne sais-tu pas que la cité ne se soucie nullement de toutes les autres ingratitudes (acharistias) et ne les juge même pas, mais qu'elle méprise ceux qui, après avoir profité de bienfaits, ne témoignent d'aucune reconnaissance; et que contre quiconque ne prodigue pas ses soins à ses parents (tis goneas mê therapeuê), la cité intente une action en justice, et que le rejetant après examen (apodokimazousa), elle ne permet pas à tel homme d'exercer une charge sous le prétexte qu'un tel sacrificateur ne saurait sacrifier avec piété au nom de la cité et qu'un tel homme ne saurait faire quoi que ce soit d'autre de façon convenable et équitable? Et, par Zeus, si quelqu'un n'entretient pas la tombe de ses parents décédés, la cité examine ce fait lors des dokimasiai des archontes (Xénophon, Les Mémorables, 2, 2, 13, [traduction Feyel 2009, p. 27]).

16 Hormis cet avertissement socratique, nous possédons un cas concret avec le procès du Contre Philon, où une génitrice malmenée par son rejeton est au cœur d'une plaidoirie visant à exclure Philon de la charge de bouleute. Il ne s'agit donc pas d'une graphê kakoseôs goneôn mais de l'examen préliminaire d'un candidat, soupçonné de kakôsis goneôn, sans pour autant que le délit soit précisément nommé. Écoutons Lysias :

Eh bien pour ma part, je suis certain que vous ne devez pas le juger autrement que ne le font ses proches. Car voici les faits, qui même s'il n'avait commis aucune autre faute, justifieraient à eux seuls son exclusion. Je passe sur les accusations dont sa mère le chargea de son vivant ; mais, si l'on en juge par la conduite qu'elle tint à ses derniers jours, il est facile de connaître quel fils ce fut. Elle n'osa pas se confier à lui pour le moment où elle serait morte; elle s'en remit à Antiphénès, qui ne lui était de rien et à qui elle donna trois mines d'argent pour sa sépulture, écartant cet homme qui était son fils. N'est-il pas évident qu'elle le savait capable de ne pas rendre, même à une mère, les derniers devoirs? Or, si une mère, qui par nature lorsqu'elle subit quelque injustice de la part de ses enfants, la supporte le mieux possible et qui, lorsqu'elle obtient quelque secours, croit en obtenir un grand, parce qu'elle examine les faits en laissant parler son cœur plutôt qu'en cherchant une preuve, a jugé qu'il pourrait profiter de sa mort, que devez-vous penser de lui? (Lysias, XXXI, Contre Philon, 20-22 [traduction Feyel 2009, p.164, légèrement modifiée]).

17 S'ajoutant à son manque de patriotisme, c'est bien un manquement indigne au respect d'une mère que le plaideur développe ici pour écarter la candidature de Philon : si la mère de Philon a préféré s'en remettre à un étranger pour ses propres funérailles, cela induit que le comportement de son fils, de son vivant, était déjà celui d'un ingrat. Il aurait pu pousser son irrespect jusqu'à la priver d'une digne sépulture, par cupidité. Or le traitement des corps morts est une des marques les plus hautes du respect envers les parents, et même si ces derniers sont de mauvais géniteurs (comme on l'a vu pour un père qui aurait prostitué son enfant). Ainsi même Électre, qui déteste sa mère au point de comploter sa mort, recouvre le cadavre de Clytemnestre (Euripide, Électre, v. 1230-1231). Philon aurait donc été un ignoble individu en ne procédant pas aux rites funéraires nécessaires lors du décès de sa mère. On pourrait cependant objecter au plaideur que si Philon était absent d'Athènes au moment des derniers instants de sa mère, cette dernière a pu vouloir préparer ses funérailles sans son fils. Et si l'on suit Christopher Carey (1989, 
p.195), il est possible finalement que la kakôsis goneôn ne couvrait pas l'absence d'organisation des funérailles par un enfant, si le parent avait choisi délibérement de s'en remettre à une tierce personne. Si Philon semble avoir entretenu des liens distendus avec sa mère, l'excès de proximité est aussi un écueil relationnel que la prohibition de l'inceste encadre strictement à Athènes.

\section{Mère interdite : l'inceste}

Dans les sources de l'Athènes classique, la notion d'inceste est couverte par le terme d' anosios gamos ou de gamos asebes (Aristophane, Les Grenouilles, v. 850 ; Sophocle, ÆEdipe à Colone, v. 945-946 ; Euripide, Électre, v. 600, 926-927 ; Eschyle, Les Suppliantes, v. 10), qui, plus largement, concerne des unions jugées impies et sacrilèges : adultères, zoophilies, nécrophilies. Parmi les délits condamnés par les agraphoi nomoi ${ }^{18}$ (absence de gratitude, manque de piété envers les dieux, irrespect envers ses parents), l'inceste demeure seul sans équivalent législatif positif ${ }^{19}$, ainsi que l'a montré Edward Harris ${ }^{20}$. En effet, on a vu que les géniteurs sont protégés par la graphê kakoseôs goneôn; la gratitude, quant à elle, est couverte par la loi sur les dettes et l'impiété envers les dieux est jugée par la graphê asebeias. Mais, concernant la répression de l'inceste, en l'absence de loi et de témoignage direct dans les sources judiciaires, seules des hypothèses sans certitude ont pu être avancées : bannissement, confiscation des biens, ou absence de sanctions (Karabélias 1989, p. 239. Sur l'inceste, voir Lévi-Strauss 1971, p. 14-29 ; Héritier 1994 ; Bonnard 2001 ; Wilgaux 2001; Wilgaux 2005b; Damet 2012, p. 195-197). Seul Platon, dans ses Lois, consacre un long paragraphe à la question de l'inceste et de son encadrement législatif : toutefois lui-même préfère laisser le traitement légal de l'inceste parmi les agraphoi nomoi. Et, pour prévenir de telles unions dans le quotidien des Athéniens, rien ne vaut l'enseignement dramaturgique des sanctions endurées par les héros ÆEdipe, Macarée et Thyeste qui, selon Platon ${ }^{21}$, se donnent la mort après leur forfait. Le crime possède intrinsèquement sa sanction. Le philosophe considère ainsi que l'inceste est un délit condamnable, que ce soit avec la mère (Ædipe), la sœur (Canacée) ou la fille (Thyeste) et que, grâce à la pédagogie tragique, les individus intériorisent une loi définissant les unions sexuelles permises, qui se passe de mise par écrit ${ }^{22}$. On notera enfin que les interdits matrimoniaux et sexuels, à Athènes, mettent bien la mère au cœur du tabou ${ }^{23}$ et ce, doublement : non seulement sont prohibées les unions entre parents et enfants, mais aussi entre demi-germains utérins, alors que les demi-germains consanguins peuvent contracter des mariages et/ou avoir des relations sexuelles (Plutarque, Vie de Thémistocle, XXXII ; Démosthène, LVII, Contre Euboulidès, 20). Il y a donc ici une nette différence entre le père et la mère, qui influe sur le statut des enfants et leurs propres relations. L'anthropologie de la parenté, notamment les récentes études relatives à l'importance du corps pour penser les liens entre individus, a ouvert aux hellénistes une voie de compréhension de cet inceste adelphique asymétrique (voir en dernier lieu Wilgaux 2011). D’après Jérôme Wilgaux (2005a), et dans le sillon de David Schneider (1984) et Françoise Héritier $(1994)^{24}$, on pourrait l'expliquer par l'importance de la matrice maternelle. Loin de l'idéologie «topique » et passive d'un Aristote, le corps de la mère n'est donc pas réductible à un seul réceptacle nourricier au rôle secondaire : il est le lieu de la création d'une identité parentale très forte, notamment entre frères et sœurs, même s'ils n'ont pas le même père. Lieu des fusions des différents sangs, la mère aurait ainsi un rôle majeur dans l'élaboration de la proximité entre parents ${ }^{25}$. Comme le résume Jérôme Wilgaux (2011, p.229), malgré la prédominance sociale et civique des relations 
patrilinéaires dans l'Athènes classique, la prohibition de l'inceste est davantage liée à la parenté naturelle et biologique qu'à la parenté légale. Si le delphus maternel transmet ainsi des humeurs identitaires qui fonderaient une parenté plus affirmée entre germains utérins, la mère est aussi un medium nécessaire pour la transmission d'une qualité indispensable à l'activité politique et à la légitimité successorale des Athéniens, la citoyenneté.

\section{1 : les mères au cœur de la transmission citoyenne}

19 La loi attribuée à Périclès en 451, en stipulant la bilatéralité nécessaire en matière de filiation citoyenne, introduit le maillon maternel dans la chaîne de fabrication et de transmission de la citoyenneté des individus. Ce rôle décisif a pour revers d'être désormais la cible potentielle des argumentations procédurières qui, dans les sources juridiques du $\mathrm{IV}^{\mathrm{e}}$ siècle, mettent souvent aux prises des adversaires, ou bien pressés de récupérer un héritage dont ils s'estiment lésés, ou bien engagés dans des règlements de compte politiques. Le droit à l'héritage est en effet intimement lié au statut de citoyen: remettre en cause le statut de la mère est un moyen de délégitimer le statut d'héritier. Ainsi que l'a très bien montré Noémie Villacèque dans un article récent (Villacèque 2014), trois types d'accusations peuvent atteindre ce but et entamer l'intégrité citoyenne d'un adversaire : avoir une mère prostituée, une mère étrangère ou une mère indigente. La fragilité de la légitimité maternelle s'explique notamment par l'absence de la mention des mères des listes des phratries et des dèmes: seuls des témoins peuvent attester de la présentation par un phratère de la nouvelle épouse. À cette occasion, le mari offre un repas, la gamêlia, aux membres de sa phratrie. La remise de la dot est aussi un moment où la présence de témoins permet de dissiper tout soupçon sur son absence et, corrélativement, sur le statut de la femme épousée : "nous invitons nos proches parce qu'il ne s'agit pas d'une affaire sans importance et que c'est la vie de nos sœurs et de nos filles que nous confions à autrui ", rappelle Démosthène (XXX, Contre Onètor I, 21). On peut encore ajouter que les fêtes de naissance sont aussi un moment de sociabilité ouverte à des familiers dont le témoignage peut être précieux, des années plus tard, si suspicion il y a sur la qualité de citoyenne de la parturiente (Gherchanoc 2015).

Malgré ces précautions, plusieurs procès du IV ${ }^{e}$ siècle nous renseignent bien sur le dossier de la fragilisation possible du statut maternel. En premier lieu, s'en prendre à la légitimité de l'épouse a pour corollaire de déconsidérer une filiation à travers la personne de la mère. Par exemple, la Succession de Pyrrhos oppose une cousine et un cousin germains : ce dernier est le fils de la sœur du défunt et il tente de prouver l'irrecevabilité successorale de Philé, qui se prétend fille légitime de Pyrrhos et réclame le patrimoine, via son mari. Le plaideur estime que Philé n'est en fait qu'une fille de courtisane, non épousée en bonne et due forme par Pyrrhos et qui l'accompagnait aux banquets (Isée, III, Succession de Pyrrhos, 14). Comme preuve à charge, le plaidant évoque l'absence de restitution de dot à la mort de Pyrrhos (Isée, III, Succession de Pyrrhos, 8-9), et envisage par conséquent l'absence de dot à l'origine (Isée, III, Succession de Pyrrhos, 28. Voir aussi Démosthène, XXX, Contre Onètor I, 12 ; Isée, II, Succession de Meneklès, 5 ; Isée, VIII, Succession de Kiron, 8). Or, un enfant issu d'une telle union, entre un citoyen et une femme qu'il n'a pas épousée kata tous nomous, ne peut pas revendiquer de succession. Les jurés ont finalement considéré qu'il y avait bien eu usurpation de statut : Philé, considérée comme la fille d'une hétaïre, a été déshéritée et reconnue publiquement comme une bâtarde (Isée, III, Succession de 
Pyrrhos, 11-15). Selon le même schéma d'accusation, on peut évoquer la Succession de Philoktémon où deux jeunes garçons affirment être les enfants légitimes de Kallipè, fille de l'Athénien Pistoxénos, contre les accusations du client d'Isée qui les présente comme les rejetons d'Alkè, une prostitutée affranchie (Isée, VI, Succession de Philoktémon, 12-14; 19-24. Voir aussi le cas bien connu du Contre Nééra). Le statut maternel est encore au cœur de la plaidoirie du Contre Euboulidès de Démosthène : Euxithéos, rayé des registres de son dème, veut prouver la citoyenneté de son père comme de sa mère. Cette dernière est attaquée cette fois-ci sur ses activités économiques : nourrice et vendeuse de rubans sur l'Agora, ses occupations professionnelles côtoient celles des sphères serviles et métèques, et l'assimilation par contamination est rapidement effectuée dans l'esprit des adversaires d'Euxithéos. Comme le rappelle David Cohen (2005, p. 34), le statut de citoyen est intimement lié aux statuts et aux jugements sociaux. On peut par conséquent nuancer les conclusions de Claude Vial (1985, p.49) qui écrivait que «dans toute société on peut douter de l'identité du père d'un individu. Rares sont les sociétés où on peut douter de celle de la mère : ce sont des sociétés, comme la société athénienne, où l'épouse reste confinée au domicile conjugal et n'y reçoit pas les amis de son mari ». C'est justement parce que certaines femmes, épouses et mères, sortent de l'oikos et croisent d'autres femmes métèques ou esclaves dans des lieux de brassage statutaire, comme peut l'être l' agora ${ }^{26}$, que la visibilité théorico-juridique des différents statuts se brouille ${ }^{27}$.

21 À l'avers de ces éléments qui soulignent la fragilité des mères (protection par l'encadrement de la kakôsis goneôn, attaques possibles du statut de citoyenne), il faut cependant mettre au jour la face dynamique et active du statut maternel. Car il y a bien des mères actrices dans le jeu judiciaire athénien, ce que certaines études " optimistes " sur le rôle des femmes dans la société athénienne, pour reprendre le qualificatif employé par Panayotis Dimakis (1989, p. 223), ont bien mis en valeur (Vial 1985; Foxhall 1995; Vial 1996 ; Sebillotte 2010 ; Damet 2014). Ces exemples permettent aussi de relativiser la question du statut des femmes, que l'on présente souvent comme "d'éternelles mineures ", constat juridique véridique, mais qui ne rend pas compte de l'existence de différents modes d'intervention des mères attiques.

\section{Mère-courage : des femmes actrices des procès}

Que les mères athéniennes, en tant que femmes, ne puissent pas ester en justice sans intermédiaire est, on l'a vu, une marque de leur personnalité juridique incomplète. Pour la plupart des spécialistes du droit attique, les femmes ne peuvent pas témoigner en personne mais les plaideurs ont la possibilité d'insérer dans leur discours un témoignage d'une citoyenne garanti par un serment prêté hors du tribunal (Just 1989, p. 33-39). La question du serment comme preuve juridique est d'autant plus intéressante pour notre propos que les témoins prêtent fréquemment serment sur la tête de leurs enfants ${ }^{28}$. Si l'on suit la formulation identique des conclusions de Claude Vial et de Barbara Levick ${ }^{29}$, il semblerait même que, dans le cas des femmes, seules des mères pouvaient prêter un serment, puisque la mention des enfants semble nécessaire. Cependant, il faut souligner que les hommes aussi intégraient fréquemment leur progéniture au serment ${ }^{30}:$ le rappel des liens parentaux masculins et féminins et l'association des enfants à celui-ci donnent davantage de crédit à une parole juridique. Notons aussi que le parjure est fréquemment associé à l'anéantissement de la maisonnée et de la descendance de celui qui ne tient pas 
parole : serment, famille et filiation sont donc bien liés, et ce pour les femmes comme pour les hommes.

Outre le serment, les questions d'héritages sont une occasion pour les femmes d'endosser un rôle d'actrices juridiques : on sait qu'à travers leurs kurioi elles pouvaient réclamer un patrimoine dont elles s'estimaient lésées. Claude Vial a très bien montré que le statut de kurios, le tuteur, n'est pas un statut pérenne ${ }^{31}$. Il s'agit justement d'un statut qui ne prend son sens que lorsque la femme agit ou souhaite agir : qu'il y ait kurios implique à la fois la dépendance des femmes mais aussi le fait qu'elles s'impliquent dans une dispute juridique (Vial 1995). Le kurios est l'incarnation juridique de la tutelle des femmes mais il est aussi une manifestation visible de leur activité. C'est précisément au cours des conflits intrafamiliaux, lorsque ceux-ci émergent à la surface du tribunal, que l'on peut, au détour des plaidoiries présentées par des hommes pour des hommes, entendre la voix de ces femmes-mères lésées ${ }^{32}$, qui défendent, dans leur combat de l'ombre, les droits de leurs enfants. Ainsi le Contre Diogiton de Lysias évoque la tenue d'un conseil de famille réuni à la demande de la fille de l'accusé : en tant que mère, elle prend fait et cause pour ses enfants contre son propre père qu'elle réprimande devant le parterre des oikeoi, car celui-ci a osé dépouillé ses petits-enfants au profit de sa nouvelle progéniture issue d'un remariage. Finalement l'affaire est menée au tribunal, exposée par un homme, le mari de la petitefille de Diogiton, qui consacre une large partie de sa plaidoirie à rapporter les accusations étayées de la fille de Diogiton, mère désespérée voulant protéger les droits de ses rejetons malmenés :

Enfin, leur mère me pria, me supplia de convoquer son père et les amis de la maison : «si peu habituée qu'elle fût, disait-elle, à parler devant des hommes, elle saurait bien, sous le coup d'une aussi grande infortune, nous exposer toute leur misère ». J'allai donc trouver Hégémon, mari de la fille de Diogiton, à qui j'exprimai mon indignation ; je parlai de la chose aux autres parents et amis, et je priai Diogiton de venir se justifier au sujet de la succession. Il refusa d'abord. Mais, finalement, ses amis l'y obligèrent, et la réunion eut lieu : «Quel cœur as-tu, lui demanda sa fille, pour te comporter ainsi à l'égard des enfants, toi, le frère de leur père, mon père à moi, leur oncle et leur grand-père? Même si tu ne rougissais devant aucun homme, tu aurais dû craindre les dieux. (...) Et maintenant tu élèves les enfants que tu as eus de ma belle-mère dans la richesse et le luxe: je ne te le reproche pas; mais les miens, tu les dépouilles, tu les chasses honteusement de la maison; de riches, tu t'appliques à en faire des gueux. » (Lysias, XXXII, Contre Diogiton, 11-18).

Là encore, si les relais judiciaires de divulgation sont forcément masculins, il faut entendre la voix de cette femme, fille et mère, qui ose finalement entamer une procédure juridique contre son propre père, action rarissime et mal perçue dans l'Athènes classique (Platon, Euthyphron. Voir Damet 2012, p. 180-182).

Un autre exemple bien connu de femme engagée dans la défense des intérêts de sa descendance est Kléoboulè, la mère de Démosthène, actrice majeure dans la préparation du procès que l'orateur lance contre ses tuteurs véreux (Hunter 1989 ; Foxhall 1995). Les Athéniens ont suivi les multiples péripéties de l'affaire et dans les différentes plaidoiries, Kléoboulè apparaît comme une femme de volonté, refusant la tutelle d'Aphobos, fils de la sœur du père de Démosthène, que le testament de son mari défunt lui imposait comme kurios et époux. Démosthène rappelle ainsi le rôle de sa mère :

Je suppose, juges, que vous êtes suffisamment instruits sur tout ce qu'ils ont volé et pillé, chacun de son côté. Vous auriez été encore mieux renseignés s'ils avaient bien voulu me rendre le testament de mon père. Tous les articles de la succession, ma mère me l'affirme, y figuraient; on y voyait aussi sur quoi les legs devaient être 
prélevés et comment mon patrimoine devait être affermé (Démosthène, XXVII, Contre Aphobos I, 40). une fortune familiale qui permettrait entre autres de marier et doter la sœur de l'orateur (Démosthène, XXVIII, Contre Aphobos II, 20-21) : ces précisions apportées par Démosthène sont assez inédites dans le sens où les sources insistent d'habitude sur le rôle du père ou $\mathrm{du}$ frère, des figures masculines, dans l'organisation économique d'un mariage et de l'octroi de la dot au futur époux. Kléoboulè présente la particularité d'être une mère veuve, une situation qui, semble-t-il, induit une indépendance certaine dans la gestion de l'oikos (Mossé 1989 ; Cudjoe 2010, p. 57-85) ${ }^{33}$. C'est précisément ce que remarque Eschine, dans sa diatribe venimeuse contre Timarque et Démosthène, en évoquant les mères affranchies par le décès de leur époux :

Démosthène, après avoir dissipé son patrimoine, se mit à parcourir la ville à la chasse de jeunes gens fortunés dont les biens étaient administrés par leurs mères, le père étant décédé. Passant sur beaucoup d'autres, je ne parlerai que de l'une de ces malheureuses victimes (Eschine, I, Contre Timarque, 170).

Ainsi que le remarquent Schaps (1979, p. 15-16 et n. 104, p. 117) et Cudjoe (2010, p. 80), cette assertion n'a pas de valeur légale en soi mais laisse entrevoir une situation de fait courante à Athènes, à savoir le rôle non négligeable de mères veuves dans la sphère économique.

\section{Conclusion : statut maternel vs statut paternel ?}

Le statut de la mère dans l'Athènes classique ne se comprend que si on le replace dans le contexte des liens étroits entre oikos et polis. La mère est une figure sociale, considérée et protégée par la cité ; elle partage avec le père les bénéfices du respect traditionnellement attaché aux parents. Il y a pourtant un traitement à la fois spécifique et commun du père et de la mère dans la sphère légale athénienne. Il est notamment intéressant de pointer le déséquilibre entre statut maternel et statut paternel, en termes de permanence et de fluidité. En effet, si un fils athénien adopté a deux pères, un biologique et un social, il n'aura toujours qu'une mère, les femmes ne pouvant pas se « fabriquer » (poiein) d'enfant. Isée nous rappelle bien que le fils adoptif n'a aucun droit sur l'héritage d'un parent de l'épouse de son père adoptif mais qu'il conserve ce droit dans sa famille maternelle $\mathrm{d}^{\prime}$ origine $\mathrm{e}^{34}$. Le statut juridiquement inamovible et indivis de la mère se retrouve dans le caractère symboliquement irremplaçable de cette même mère, ce que l'on peut discerner dans les discours dépréciant le statut de belle-mère (Homère, Iliade, v. 388-390 ; Hésiode, Les Travaux et les Jours, v. 825 ; Hérodote, IV, 154 ; Platon, Les Lois, XI, 930b ; Aristote, Génération des animaux, III-9, 759a36; Euripide, Alceste, v. 304-306; Ion, v. 1024-1025; Hippolyte, v. 960 ; Égée, fr. 16. Voir Watson 1995 ; Damet 2012, p. 107-111), mêtruia, nouvelle épouse d'un père. Comme l'écrivait déjà Giulia Sissa (2005, p. 58), « entre une mère et la progéniture issue de son corps, il y a une familiarité aussi irremplaçable qu'anonyme. Ainsi, du côté paternel, il est possible qu'un enfant soit détaché du lignage et il est admis qu'un demi-frère épouse une demi-sœur. La lignée utérine est au contraire le lieu d'une transmission intarissable et, aussi, de l'inceste adelphique ». Sur le même principe, alors qu'il est possible pour un père athénien de renier son fils, par le biais de la procédure de l' apokêruxis (Beauchet 1976 [1897], II, p. 128-146; Wurm 1972 ; MacDowell 1978; Damet 2012, p. 190-195), il n'est pas prévu par la loi attique qu'une mère puisse se décharger de 
sa maternité sociale. Seule Clytemnestre, mère tragique pleine d'andreia virile ${ }^{35}$, rejette ( ekballein) sa fille Électre (Sophocle, Électre, v. 590 et Euripide, Électre, v. 61), qui considère cette première davantage comme une maîtresse que comme une mère. Dans le cadre des relations père/fils, on peut établir un parallèle entre l'apokêruxis, la graphê paranoias et la graphê argias : ces deux dernières procédures pouvaient être utilisées contre un père qui dilapidait le patrimoine familial, perdant la raison au point de fragiliser l'équilibre économique de l'oikos (Xénophon, Les Mémorables, I, 2, 49 ; [Aristote], Constitution d'Athènes, LVII, 6 ; Aristophane, Les Nuées, v. 845 ; Platon, Les Lois, XI, 929e. Voir Harrison 1968-1971, t. 1, p. 80 ; Cantarella 2011, p. 337 ; Damet 2012, p. 183-184, 191). Si les pères et les fils d'Athènes ont à disposition des armes juridiques pointées l'un contre l'autre, les mères sont-elles exclues de ces potentialités conflictuelles? Si l'on envisage que des mères athéniennes, notamment veuves, pouvaient bien gérer un patrimoine avec une certaine autonomie, ces dernières échappaient-elles à la surveillance des gestions de fortune induite par la graphê paranoias et la graphê argias $^{36}$ ? En matière de statut, il y a bien une difficulté à déceler les charges pesant sur la mauvaise mère, un impensé des sources juridiques athéniennes.

\section{BIBLIOGRAPHIE}

Adam-Magnissali S. (2009), «Droit et altérité dans le monde ancien : le cas des mineurs dans l'Athènes classique ", dans Harris E. et Thür G. éd., Symposion 2007. Vorträge zur griechischen und hellenistischen Rechtsgeschichte, Vienne, p. 145-160.

Adam-Magnissali S. et Cudjoe R. (2010), « Family law in [Demosthenes] 43: Against Makartatos, 75 », EHHD 42, p. 67-93.

Avotins I. (2004), « Athenaion Politeia 56.6 and the protection of the weak », CQ 54-2, p. 461-469.

Azoulay V. (2004), Xénophon et les grâces du pouvoir. De la charis au charisme, Paris.

Azoulay V. et Damet A. (2014), « Paroles menaçantes et mots interdits en Grèce ancienne : approches anthropologiques et juridiques », Cahiers « Mondes anciens » 5 [En ligne]. URL : http:// mondesanciens.revues.org/1211; DOI : 10.4000/mondesanciens.1211

Barraud G. (1954), « La puériculture et l'eugénisme chez les Grecs », BAGB 1, p. 70-76.

Beauchet L. (1976 [1897]), Histoire du droit privé de la République athénienne, New York.

Beaumont L. (2012), Childhood in Ancient Athens: Iconography and Social History, Londres.

Bonfante L. (1997), « Nursing mothers in classical arts », dans Koloski-Ostrow A.-O. et Lyons C. éd., Naked truths: Women, Sexuality, and Gender in Classical Art and Archaeology, Londres, p. 174-196.

Bonnard J.-B. (2001), « Phèdre sans inceste. À propos de la théorie de l'inceste du deuxième type et de ses applications en histoire grecque », RH 304, p. 77-107.

- (2003), « Un aspect positif de la puissance paternelle : la fabrication du citoyen », Mètis N.S. 1, p. 69-93.

- (2004), Le complexe de Zeus. Représentation de la paternité en Grèce ancienne, Paris. 
Brulé P. (2006), « La parenté selon Zeus », dans A. Bresson et al. éd., Parenté et société dans le monde grec de l'Antiquité à l'âge moderne, Bordeaux, 2006, p. 97-119.

Carey C. (1989), Lysias. Selected Speeches, Cambridge.

Cantarella E. (2010), « Fathers and sons in Athenian law and society », dans Thür G. éd., Symposion 2009. Vorträge zur griechischen und hellenistischen Rechtsgeschichte, Vienne, p. 1-22.

- (2011), « Greek law and the family », dans Rawson B. éd., A Companion to Families in the Greek and Roman Worlds, Chichester, p. 331-345.

Christ M. (2006), The Bad Citizen in Classical Athens, Cambridge.

Cohen D. (2005), « Women in public: gender, citizenship, and social status in classical Athens ", dans Gagarin M. et Wallace R. éd., Symposion 2001. Vorträge zur griechischen und hellenistischen Rechtsgeschichte, Vienne, p. 33-45.

Cudjoe R. (2010), The Social and Legal Position of Widows and Orphans in Classical Athens, Athènes.

Damet A. (2011), « Le sein et le couteau. L'ambiguïté de l'amour maternel dans l'Athènes classique », Clio. HFS 34, p. 17-40.

- (2012), La septième porte. Les conflits familiaux dans l'Athènes classique, Paris.

- (2014), « La domination masculine dans l'Athènes classique et sa remise en cause dans les crises intrafamiliales », Siècles 35-36 [En ligne]. URL : http://siecles.revues.org/1503

Devereux G. (2006), Les rêves dans la tragédie grecque, Paris.

Dimakis P. (1989), «Quelques remarques à propos de la position juridique des Athéniennes à l'époque classique (Tentatives en vue de l'effondrement d'un mythe) », dans Thür G. éd., Symposion 1985. Vorträge zur griechischen und hellenistischen Rechtsgeschichte, Cologne, p. 221-231.

De Schutter X. (1991), « Piété et impiété filiales en Grèce », Kernos 4, p. 219-243.

Dorion L.-A. (2006), « Socrate et l'utilité de l'amitié », Revue du MAUSS 27, p. 269-288.

Dubois C. (2012), « Des objets pour les bébés ? Le dépôt de matériel dans les sépultures d'enfants en bas âge du monde grec (VIII ${ }^{\mathrm{e}}-\mathrm{IV}^{\mathrm{e}}$ s. av. notre ère) », dans Hermary A. et Dubois C. éd., L'enfant et la mort dans l'Antiquité. III, Le matériel associé aux tombes d'enfants, Paris, Aix-en-Provence, p. 329-342.

Eidinow E. (2007), « Why the Athenians Began to Curse », dans Osborne R. éd., Debating the Athenian Cultural Revolution: Art, Literature, Philosophy and Politics, c. 430-380 BC, Cambridge, p. 44-71.

Feyel C. (2009), Dokimasía : la place et le rôle de l'examen préliminaire dans les institutions des cités grecques, Paris.

Fialho M. do C. (2010), « Paidotrophia and gêrotrophia: reciprocity and disruption in Attic tragedy », dans Harris E. et al. éd., Law and Drama in Ancient Greece, Londres, p. 108-121.

Fisher N. (1992), Hybris : A Study in the Values of Honour and Shame in Ancient Greece, Warminster.

Foxhall L. (1996), «The law and the lady: women and legal proceedings in classical Athens », dans Foxhall L. et Lewis A. éd., Greek Law in Its Political Setting: Justifications Not Justice, Oxford, p. 133-152.

Gagliardi L. (2005), «The Athenian Procedure of "dokimasia" of Orators. A Response to Douglas M. MacDowell », dans Gagarin M. et Wallace R. éd., Symposion 2001. Vorträge zur griechischen und hellenistischen Rechtsgeschichte, Vienne, p. 89-97. 
Gherchanoc F. (1998), « Le lien filial dans l'Athènes classique. Pratiques et acteurs de sa reconnaissance », Mètis XIII, p. 313-344.

- (2012), L'oikos en fête. Célébrations familiales et sociabilité en Grèce ancienne, Paris.

- (2015), « Naissance et rites de passage dans l'Athènes classique », dans López R.M.C. éd., Pariry nacer en el Mediterráneo antiguo. Entre el mito y la historia, Vigo (à paraître).

Harrison A.R.W. (1968-1971), The Law of Athens, 2 tomes, Oxford.

Harris E. (1999), « The penalty for frivolous prosecution in Athenian Law », Dike 2, p. 23-142.

- (2006), « Antigone the Lawyer, or the Ambiguities of Nomos », dans Harris E. éd., Democracy and the Rule of Law in Classical Athens: Essays on Law, Society, and Politics, Cambridge, p. 41-80.

Héritier F. (1994), « L'inceste dans les textes de la Grèce classique et post-classique », Mètis IX-X, p. 99-115.

Humphreys S. (1994), « Le mariage entre parents dans l'Athènes classique », dans Bonte P. éd., Épouser au plus proche, Paris, p. 31-58.

Hunter V. (1989), « Women's authority in classical Athens », EMC 8-1, p. 39-48.

Kamen D. (2013), Status in Classical Athens, Oxford.

Kapparis K. (1995), « When were the Athenian adultery laws introduced ? », RIDA 42, p. 97-122.

Karabélias E. (1989), « Inceste, mariage et stratégies matrimoniales dans l'Athènes classique », dans Thür G. (éd.), Symposion 1985. Vorträge zur griechischen und hellenistischen Rechtsgeschichte, p. 233-251.

Karabélias E. (2002), L'épiclérat attique, Athènes.

Larran F. (2014), « Théomnestos au tribunal ou l'injure comme arme du citoyen », Cahiers

«Mondes anciens » 5 [En ligne]. URL : http://mondesanciens.revues.org/1241 ; DOI : 10.4000/ mondesanciens.1241

Leão D. (2011), « Paidotrophia et gerotrophia dans les lois de Solon », RHD 89-4, p. 457-472.

Lee E.N. et al. éd. (1973), Exegesis and Argument. Studies in Greek Philosophy presented to G. Vlastos, Assen, p. 70-104.

Lévi-Strauss C. (1971), Les structures élémentaires de la parenté, Paris.

Levick B. (2012), « Women and Law », dans James S. L. et Dillon S. éd., A Companion to Women in the Ancient World, Malden-Oxford, p. 96-106.

Loraux N. (1986), « Matrem nudam », ÉdT 11, p. 90-102.

MacDowell D. (1978), The Law in Classical Athens, Ithaca.

- (2005), « The Athenian procedure of Dokimasia of Orators ", dans Gagarin M. et Wallace R. éd., Symposion 2001.Vorträge zur griechischen und hellenistischen Rechtsgeschichte, Vienne, p. 79-87.

Mossé C. (1989), « Le Contre Spoudias de Démosthène et les droits économiques des femmes d'Athènes au IV siècle », dans Thür G. éd., Symposion 1985. Vorträge zur griechischen und hellenistischen Rechtsgeschichte, p. 215-221.

Morgan J. (2011), «Families and religion in classical Greece », dans Rawson B. éd., A Companion to Families in the Greek and Roman Worlds, Chichester, p. 447-464.

Ostwald M. (1973), « Was there a Concept of agraphoi nomoi in Classical Greece? », dans Lee et al. 1973. 
Patterson C. (1986), « Hai Attikai : the Other Athenians », Helios 13, p. 49-67.

Petersen L. et Salzman-Mitchell P. (2012), Mothering and Motherhood in ancient Greece and Rome, Austin.

Roux O. (2009), « Parenté hippocratique et parenté aristotélicienne. Quelques réflexions sur les théories biologiques de la Grèce ancienne ", Pallas 79, p. 307-322.

Rubinstein L. (1993), Adoption in IV. Century Athens, Copenhague.

Ruschenbusch E. (2010), Solon : Das Gesetzeswerk-Fragmente, Historia 215.

Salzman-Mitchell P. (2012), « Tenderness or Taboo: Images of Breast-Feeding Mothers in Greek and Latin Literature », dans Petersen et Salzman-Mitchell 2012, p. 141-164.

Schaps D. (1979), Economic Rights of Women in Ancient Greece, New York.

Schneider D. (1984), A Critique of the Study of Kinship, Ann Arbor.

Sebillotte V. (2010), « La démocratie athénienne et les femmes », dans Riot-Sarcey M. éd., De la différence des sexes. Le genre en histoire, Paris, p. 23-48.

Sissa G. (2005), « La famille dans la cité grecque, $V^{\mathrm{e}}-\mathrm{IV}$ e siècles av. J.-C. », dans Rousselle A. et al. éd., La famille dans la Grèce antique et à Rome, Bruxelles, p. 12-64.

Siron N. (2014), «L'autorité du témoignage : où est le genre ? Femmes et hommes à la barre dans les procès à Athènes au IV ${ }^{\mathrm{e}}$ siècle avant $\mathrm{J} .-\mathrm{C}$. " , $14^{\mathrm{e}}$ congrès de la FIEC, Bordeaux.

Vernant J.-P. (1987), « L'individu dans la cité », dans Veyne P. éd., Sur l'individu, Paris, p. 20-37.

Vial C. (1985), « La femme athénienne vue par les orateurs », dans Verilhac A.-M. éd., La femme dans le monde méditerranéen, 1 : Antiquité, Lyon, p. 47-60.

Vial C. (1995), «Statut et subordination », dans Cavalier O. éd., Silence et fureur. La femme et le mariage en Grèce, Avignon, p. 340-357.

Vilatte S. (1991), « La nourrice grecque », AC 60, p. 5-28.

Villacèque N. (2014), « Ta mère ! Insulte et généalogie à la tribune démocratique », Cahiers «Mondes anciens » 5 [En ligne]. URL : http://mondesanciens.revues.org/1242 ; DOI : 10.4000/ mondesanciens. 1242

Watson P. (1995), Ancient Stepmothers: Myth, Misogyny, and Reality, Leyde.

Wilgaux J. (2001), « Entre inceste et échange : réflexions sur le modèle matrimonial athénien », L'Homme 158-159, p. 659-676.

Wilgaux J. (2005a), « David Schneider en Attique : le sang, le sperme dans les représentations de la parenté en Grèce ancienne », Incidence 1, p. 75-90.

Wilgaux J. (2005b), « Inceste et barbarie en Grèce ancienne », Cahiers KUBABA 7, p. 267-270.

Wilgaux J. (2011), « Consubstantiality, Incest, and Kinship in Ancient Greece », dans Rawson B., A Companion to Families in the Greek and Roman Worlds, Oxford, p. 217-230.

\section{NOTES}

1. Sur le traitement de la figure maternelle dans le corpus gortynien, voir la contribution de Maude Lajeunesse dans ce volume. 
2. Finley 1981, p. 217 : «Il faut envisager le statut comme un faisceau de privilèges, de capacités, etc., et par conséquent concevoir la définition de tout statut particulier, ou du statut d'un individu précis, en termes de possession et de localisation, chez cet individu, de tel ou tel élément du faisceau. »

3. Traduction CUF légèrement modifiée. Sauf indication contraire, les traductions adoptées sont celles de la Collection des Universités de France (CUF), aux Belles Lettres.

4. Le statut épicléral pose lui-même la question du statut maternel : selon E. Karabélias (2002, p. 145-158), c'est justement lorsqu'une potentielle épiclère a des enfants, garçons ou filles, qu'elle ne peut plus être réclamée par le parent ayant-droit. "L'aphairesis de la femme mariée devenue ultérieurement épiclère» serait donc impossible en cas de maternité avérée. D'une part, concernant l'existence de fils, « la parenté par les femmes et la succession par souches, dans les limites du privilège de masculinité constituaient des principes irréfutables pour le droit attique. Le petit-fils donc du défunt, issu de sa fille, trouvait sa pleine vocation successorale (...) Il est sans aucun doute héritier de son aïeul maternel et exclut tout autre collatéral successible» (p. 154). D'autre part, concernant l'existence d'une descendance féminine, « la fille du défunt avait pleine vocation successorale dans les structures de l'oikos grec. En conséquence nous ne voyons pas d'obstacle à admettre l'impossibilité de l'aphairesis de l'épiclère mariée ayant des filles seules » (p. 155).

5. Sur la gratitude envers les parents, voir Homère, Odyssée, II, 130-131; Hésiode, Les travaux et les jours, v. 185-188 ; Lycurgue, I, Contre Léocrate, 94 ; Platon, Lois, IV, 717b-c ; Aristote, Éthique à Nicomaque, 1163b ; Plutarque, Sur l'amour fraternel, 479f-480a.

6. Sur l'humeur de Xanthippe, voir Platon, Banquet, II, 9-10; Xénophon, Mémorables, II, 2, 7 («Pour ma part, répondit-il, je crois que la plus insupportable [humeur à supporter] est celle d'une mère, en tout cas d'une mère comme la mienne. »).

7. Diogène Laërce, II, 29 : «Il fit changer d'avis aussi Lamproklès, son fils, qui était en colère contre sa mère, comme Xénophon le dit quelque part. » (trad. M.-O. Goulon Cazé, Pochothèque).

8. Ceci explique qu'đEdipe considère ses filles Antigone et Ismène comme des «fils » car ce sont elles qui soutiennent leur père dans son exil (Sophocle, EEdipe à Colone, v. 444-447 et 1360-1374). Polynice et Étéocle agissent, eux, selon les «coutumes égyptiennes » (v. 337) car, en Égypte, ce sont les filles et non les fils qui sont dans l'obligation légale de nourrir leurs parents (Hérodote, II, 35, 18-20).

9. C. Vial (1985, p. 59) : «Ce que les plaidoyers nous montrent, ce sont les archétypes élaborés par la société masculine. Vue par les hommes, la Femme est avant tout la Mère. Elle est celle qui permet la continuation de la lignée ; qui permet la légitimité des enfants ; qui permet d'hériter, de jouir des droits civiques, d'avoir des parents par le sang et par l'alliance. Elle est aussi celle qui aime et protège ses enfants ; qui leur vient en aide, s'il le faut, quand leur père n'est plus là ; elle est celle sur qui les enfants peuvent compter. Il y a de mauvais pères dans les plaidoyers; hormis la femme adultère qui fait pleurer son bébé pour berner son mari, il n'y a pas de mauvaises mères. "

10. Loraux 1986 ; Vilatte 1991 ; Damet 2011 ; Beaumont 2012, p. 52-61. Sur l'allaitement maternel (non exhaustif) : Hymne à Hermès, v. 267 ; Homère, Odyssée, II, v. 447-449 ; Iliade, XXII, v. 79-84 ; Eschyle, Les Choéphores, v. 896-898; Lysias, I, Sur le meurtre d'Érastosthène, 9-10; Xénophon, Les Mémorables, II, 2, 5.

11. Sur les femmes tragiques et les mauvaises mères, Foley 2001 ; Damet 2012.

12. Sophocle, Électre, v. 187-192 : «Je me consume ici, comme une réfugiée que nul ne considère, je suis servante au palais de mon père, dans l'appareil indigne où tu me vois, et faisant le siège de tables qui sont toujours vides (kenais) pour moi. »

13. Aux vers 523-550 des Choéphores, le Coryphée rapporte à Oreste le cauchemar de sa mère, qui préfigure le meurtre de Clytemnestre par ce dernier. Elle aurait enfanté un serpent à qui elle donne le sein ; elle est alors mordue au mamelon et au lait se mêle un caillot de sang. Or, dans la 
pièce précédant les Choéphores, l'Agamemnon, Oreste est comparé à un lionceau privé du lait de sa mère (agalakton, v. 718) et la nourrice des Choéphores, aux vers 749-762, explique que c'est elle qui s'est occupée d'Oreste à sa naissance, remplaçant Clytemnestre, mère défaillante. Clytemnestre n'a pas nourri son fils et pourtant elle utilise cet argument, affectif, corporel et symbolique pour tenter de l'amadouer au moment du matricide (Eschyle, Les Choéphores, v. 898-908 ; voir Loraux 1986 ; Damet 2011). Ainsi que l'a brillamment démontré Georges Devereux (2006, p. 273-331), le songe de Clytemnestre n'est pas « une distorsion cauchemardesque des souvenirs de l'allaitement d'Oreste » mais bien un aveu d'absence d'allaitement, incarné par la mise au monde d'un serpent de la culpabilité et de la vengeance. Dans son rêve, les deux actes coupables de Clytemnestre, mauvaise mère (qui n'a pas allaité Oreste) et mauvaise épouse (qui a tué Agamemnon), se mélangent, comme le sang et le lait.

14. [Démosthène], LXIX, Contre Nééra, 87 ; Eschine, I, Contre Timarque, 183. Ces dispositions sur la répression de la moicheia auraient été introduites à la suite de la loi de Périclès sur la citoyenneté. Voir Kapparis 1995.

15. La législation platonicienne contient aussi un arsenal punitif contre les mauvais fils : une partie des agressions que les parents de Magnésie subissent, voies de faits ou injures, recoupe la désignation attique de kakôsis goneôn, que Platon nomme aikia goneôn (Lois, XI, 932a). L'exil à perpétuité est prévu pour celui qui a osé porter la main sur un père, une mère, ou des aïeux et qui les violente et les maltraite (Lois, IX, 879b6-882c4).

16. [Aristote], Constitution d'Athènes, LVI, 6. Voir Avotins 2004 : ce dernier démontre qu'il existait un recours possible à deux types de poursuite pour kakôsis gonêon, la graphê et l'eisangélie, toutes deux sans danger pécunier pour l'initiateur.

17. Lysias, XIII, Contre Agoratos, 91. Selon Euripide, Alceste, v. 679; [Démosthène], XL, Contre Boeotos II, 45 ; Hésiode, Les Travaux et les Jours, v. 183 et 331, les insultes et les mauvaises paroles pourraient aussi tomber sous le coup de la kakôsis goneôn, en tant qu'outrage, hubris. Voir Fisher 1992, p. 92.

18. Sur le concept de loi non écrite, voir Ostwald 1973 ; Harris 2006.

19. En France, il aura fallu attendre la loi du 8 février 2010 pour voir l'inceste inscrit dans la législation (Loi $n^{\circ}$ 2010-121 du 8 février 2010 tendant à inscrire l'inceste commis sur les mineurs dans le code pénal et à améliorer la détection et la prise en charge des victimes d'actes incestueux). La législation n'en faisait jusqu'ici qu'une circonstance aggravante pour les viols et les autres agressions sexuelles.

20. Platon, Les Lois, VIII, 838c. Pour Xénophon, Les Mémorables, IV, 4, 19-23, le châtiment des unions incestueuses entre parents et enfants est compris dans le produit même de cette relation, une descendance affaiblie et dégénérée. Selon une vision très eugéniste, les partenaires ne sont en effet pas dans la bonne fourchette d'âge pour une procréation optimale.

21. Que l'issue de ces unions incestueuses ait été la mort est une exagération de Platon, probablement dans le souci d'effrayer davantage celui qui pourrait avoir ces désirs insensés : l'œdipe sophocléen s'est crevé les yeux, Thyeste n'est pas mort de son inceste, mais son destin est imbriqué dans la malédiction familiale des Atrides, et Macarée seul se donne la mort, non pas tant par culpabilité, mais parce que sa sœur s'est elle-même suicidée.

22. Les parents interdits sont donc peu nombreux. Un Athénien peut épouser sa demi-sœur paternelle, sa nièce et tous les types de cousines : Euripide, Andromaque, v. 173-176; Xénophon, Cyropédie, V, 1, 10 ; Xénophon, Les Mémorables, 4.4.19-23 ; Platon, République, V, 461b ; Platon, Les Lois, VIII, 838a-d ; Démosthène, LVII, Contre Euboulidès, 20 ; Plutarque, Vie de Thémistocle, XXXII. Voir Wilgaux 2001.

23. Le motif de la mère, tabou sexuel, est aussi utilisé pour expliquer, en partie, l'absence des scènes d'allaitement sur les vases attiques (Bonfante 1997, p. 188 ; Salzman-Mitchell 2012). Comme le résume Nicole Loraux (1986, p. 100), il serait vain, quand les mères dévoilent leur sein pour allaiter, de vouloir séparer deux images psychiques, « la mère de la femme désirable ». 
24. Pour d'autres hypothèses, plus économiques ou sociologiques, voir Karabélias 1989 ; Humphreys 1994.

25. Wilgaux 2011, p. 228 : «Women, in the Greek representations, are considered as the place where male bodily susbtances meet, and mix and take effect. The female womb, which is transformed and impregnated by these successive relationships, become able to create identity, to turn a plurality of people into one single entity. Consequently, children born of the same mother could be considered as closer than children born of the same father, not because of a privileged maternal bond but because the maternal womb is the place where fusion occurs. The main point is not that she gives her own blood but that she blends the different bloods. »

26. On pensera aussi aux chantiers où citoyens ouvriers, manœuvres métèques, esclaves se mêlent parmi les blocs de marbre. Voir les comptes du chantier de l'Érechteion, IG I ${ }^{3}$ 474-479.

27. C'est bien ce qu'un pseudo-Xénophon bougonnant regrette dans l'Athènes classique: les brouillages entre les statuts (Constitution des Athéniens, 10-11).

28. Femmes prêtant serment sur la tête de leurs enfants: Lysias, XXXII, Contre Diogiton, 13; Démosthène, XXIX, Contre Aphobos III, 26, 33. Femmes prêtant serment sans faire mention de leurs enfants mais dont on sait qu'elles sont mères: Démosthène, XXXIX, Contre Boeotos I, 3-4 et II , 10-11; Démosthène, LV, Contre Calliclès, 57 ; Isée, XII, Pour Euphiletos, 12.

29. Vial 1985, p. 52 : «on peut en justice établir un fait par trois types de déclarations : les témoignages des hommes libres, les déclarations des esclaves sous la torture et les serments sur la tête de leurs enfants prononcés par les femmes libres dans un sanctuaire ». Voir aussi Levick 2012, p. 103 : «In Athens, a woman could testify if she were free and took an oath on the heads of her children. »

30. Démosthène, XXIX, Contre Aphobos III, 52-54 ; Démosthène, XXIII, Contre Aristocrate, 67-68 ; [Démosthène], Contre Conon, 38, 40 ; Eschine, III, Contre Ctésiphon, 77 ; Dinarque, I, Contre Démosthène, 71. Je remercie Nicolas Siron (2014) pour nos échanges autour de cette question du genre dans la validité des témoignages.

31. Vial 1996, p. 346. Concernant le kurios, on rappellera que la procédure de divorce initiée par l'épouse semble se passer de l'intermédiaire du tuteur: l'épouse doit se présenter en personne devant le magistrat compétent pour déposer sa demande. Le droit athénien envisage donc des situations où il est logique que le kurios ne soit pas présent. Voir Cohn-Haft 1995 ; Norena 1998.

32. Des femmes de justice qui peuvent aussi être des sœurs : voir Mossé 1989; Dimakis 1989 ; Foxhall 1996 ; Damet 2014.

33. Voir aussi Démosthène, XLI, Contre Spoudias, 8-11.

34. Isée, VII, Succession d'Apollodore, 25 : «L'adoption ne détache pas l'enfant de la mère : elle reste toujours la mère, soit que l'enfant demeure dans la maison paternelle, soit que l'adoption l'en fasse sortir. "

35. Eschyle, Agamemnon, v. 10-11: «Car c'est ainsi que règne, dans sa virile détermination ( androboulon), un cœur de femme qui attend. » L'amant de Clytemnestre, Égisthe, est, pour sa part, féminisé (Eschyle, Agamemnon, v. 1625-1627) : «Femme (gunai)! tu es restée à la maison comme ménagère en attendant le retour du combat des hommes. Tu as déshonoré la couche d'un homme et tu as comploté ce meurtre d'un homme qui était chef d'armée. » Voir Damet 2014.

36. Une femme pouvait être accusée dans le cadre d'une graphê xenias ou une dikê phonou. Comme le résume Esther Eidinow, (2007, p. 63, n. 70), puisque la "citoyenne " athénienne ne possède officiellement pas de bien propre, elle ne peut pas être condamnée lors de procès réglés par le paiement d'amendes ou de réparation de dommages. Dans le cas de la graphê paranoias, en l'absence de sources directes et de cas avérés, il est difficile de savoir ce qu'encourrait le coupable (une mise sous tutelle?). 


\section{RÉSUMÉS}

Les sources juridiques athéniennes permettent de saisir la figure maternelle comme un sujet de droits : être mère signifie profiter de la protection encadrée par la loi et accordée aux veuves, aux épiclères et aux personnes âgées. La mère est en outre au cœur de la transmission citoyenne depuis la loi de Périclès et toutes les docimasies athéniennes lui accordent une place importante. À Athènes, la prohibition légale et culturelle de l'inceste pose la mère comme sujet principal des interdits. Il est, en revanche, plus difficile de définir les devoirs qui incombent au sujet juridique maternel, même dans le domaine de la trophê, souvent mise en avant par les textes évoquant le rôle attendu des génitrices. On trouvera enfin des cas avérés de procès où la mère, notamment veuve, a bien un rôle actif dans le déroulement des conflits.

The study of Athenian law is useful to portray mothers as a subject of rights : the Athenian state protects his widows, epikleroi and older people. The Pericles law on citizenship puts mothers at the heart of legitimacy and so do all dokimasiai. The maternal womb is moreover important to understand the limits of the Athenian prohibiton of incest. The duties of Attic mothers are more difficult to grasp, even about trophe. Finally, trials from Classical Athens provide some maternal figures, mostly widows, who seem to control the economical and juridical situations.

\section{INDEX}

Mots-clés : mère, droit, Athènes, docimasie, inceste, obligation de nourriture, citoyenneté Keywords : motherhood, athenian law, dokimasia, incest, gerotrophia, citizenship

\section{AUTEUR}

\section{AURÉLIE DAMET}

Université Paris 1 Panthéon-Sorbonne, ANHIMA - UMR8210 